\title{
3DMSNET: 3D CNN Based Brain MRI Segmentation
}

\author{
Junaid Ahmad, Bhanu Bhaskar, Haresh Seetharaman, Ajay Kumar, J. Arunnehru
}

\begin{abstract}
Segmentation of the brain images has become an important task to analyze the abnormality in infants. Automatic methods are important as the infant brain growth has to be tracked and it is almost impossible for an individual to manually segment the MRI data on particular intervals. The manual segmentation tasks are time-consuming and require highly skilled professionals to segment images. Automatic segmentation methods have gained huge support for segmenting MRI images. Several segmentation methods lack accuracies due to nearest neighbor or self-similarity problems. The CNNs have outperformed the traditional methods and are proving to be more reliable day by day. The proposed method is a patch-based method which uses 3DMSnet (3D Multi-Scale Network) for segmentation. The model is evaluated on BrainWeb and other publicly available datasets.
\end{abstract}

Index Terms- segmentation, nearest-neighbor, self-similarity, patch-based, BrainWeb, CNN, MRI, 3DMSnet

\section{INTRODUCTION}

The basic approach of the segmentation methods is to label the MRI data into GM (gray matter), WM (White matter) and CSF (Cerebrospinal Fluid). There are several state-of-art methods which are used to segment the images. The intensity-based methods [1] aim to classify individual pixels/voxels into the 3 classes mentioned above. These approaches also make use of probabilistic atlases to enhance the classification. Region growing methods [2] which start from a single pixel or a small group and starts expanding comparing the intensities of the neighboring voxels/pixels. This method works best for the tissue level segmentation of the brain image for region-based lesion or vessel segmentation tasks. Clustering methods are used to cluster the pixels/voxels with similar intensities and not require labels for training. The most commonly used methods of clustering are the k-means, Expectation-maximization [3] and FCM (fuzzy C-means) [4]. The atlas-based methods of segmentation are very powerful as the atlases can be used as prior knowledge for segmentation. In order to use these atlases for the segmentation process, it has to be properly inclined with the target image. The method of overlaying the

Revised Version Manuscript Received on Jun 20, 2019.

Junaid Ahmad, Department of Computer Science, SRM Institute of Science \& Technology, Chennai, India.

Bhanu Bhaskar Kotagiri, Department of Computer Science, SRM Institute of Science \& Technology, Chennai, India.

Haresh Seetharaman, Department of Computer Science, SRM Institute of Science \& Technology, Chennai, India.

Ajay Kumar, Department of Computer Science, SRM Institute of Science \& Technology, Chennai, India.

J. Arunnehru, Department of Computer Science, SRM Institute of Science $\&$ Technology, Chennai, India. atlases with the target images is called image registration. The registration facilitates the spatial alignment of the corresponding features on the target image. The registration of a non-growing part can be done using rigid registration otherwise a non-rigid registration must be used $[5,6]$. Classification methods use the labels as prior knowledge. The image features are basically the pixel intensity or textures. The most basic classifier is the k-NN classifier [7] where pixel by pixel classification is done in the classes mentioned in the training data considering the least possible intensity difference. The k-NN method usually performs best with large training data. Segmentation methods were further improved with the use of Convolutional Neural Networks (CNN). CNNs have performed better in object recognition, segmentation, and scene understanding. CNNs have been incorporated in the

brain segmentation process and accurate results have been achieved. Moeskops et al. [8] uses the multi-scale CNN to maintain spatial consistency as well as intensity characteristics. This paper makes use of patch-based CNN model to segment the brain images into WM, GM, and CSF. It also uses a multi-scale CNN like [8] for spatial consistency. The experiment is conducted on the Simulated Brain Web Data [9] which provides a custom simulation of Real MRI data and their corresponding labels. It also provides ground truth data which can be used for testing of the trained model.

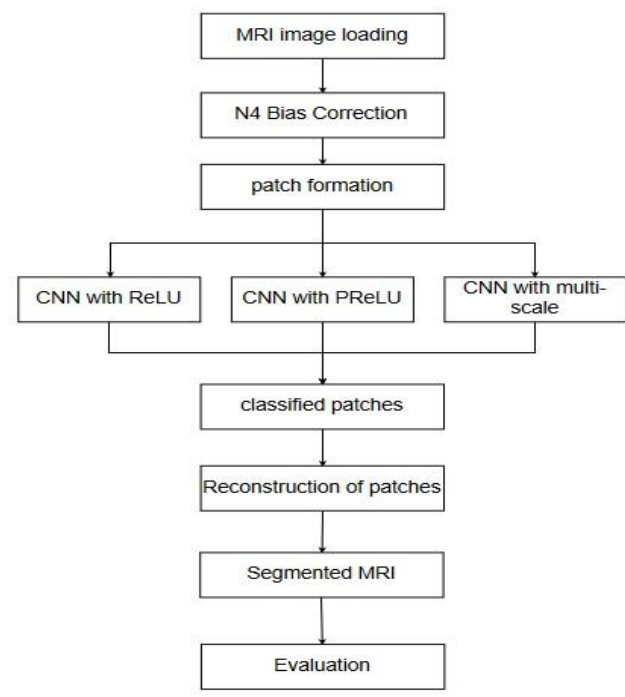

Figure 1: Overview of the proposed work

\section{METHOD}

The initial step of the segmentation is processing of the MRI data into efficiently usable form. The MRI data consist of 3D data 
which cannot be directly passed into a model for training or testing.

The data needs to be filtered, divided and normalized. In case of other methods, the data need to be registered using rigid/non-rigid registration to maintain the proper alignment of the features. The pre-processing steps involve bias correction and building patch sets. After preprocessing, the model is trained and tested for accuracy. The labeled patches are then reconstructed into the complete 3D image. The reconstructed images are then used for evaluation and performance analysis. Fig. 1 gives an overview of the complete process.

\section{A. Bias Field Correction}

Bias fields are low-frequency signals produced by the MRI machines which adds an undesirable noise to the MRI. Bias fields are caused due to the magnetic field variations caused due to the interaction with human body. The Bias field depends on the strength of the magnetic field used. The stronger the magnetic field higher the bias field. It should be removed as it would significantly reduce the accuracy of segmentation or classification if not removed. This paper uses the N4 bias correction [10] method for bias field removal. Fig. 2 shows the MRI slice, Bias mask and the Bias corrected image.

\section{B. Patch Formation}

The MRI image cannot be directly used for training any model. The volume of the MRI is too high to be directly fed to neural networks. Hence, the volumes are segmented into patches of a fixed size. After experimenting the accuracy on different patch sizes, we chose the patch size of $25 \times 25 \times 25$ as the input data and patch sizes of $15 \times 15 \times 15$ are obtained at the output end. Other works like Kamnitsas et al [11] derives that the patch sizes above $25 \times 25 \times 25$ reduce the performance of the classification. Since the output layer gives out patches
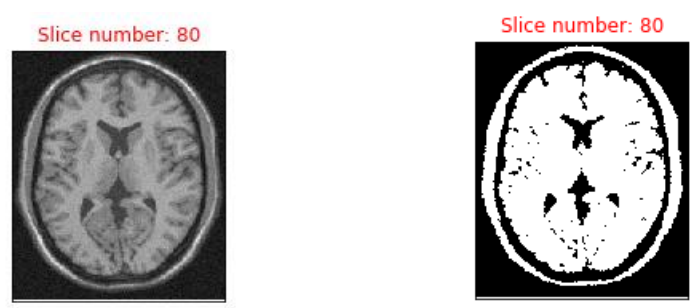

(a) Before Bias Correction

(b) Bias Mask

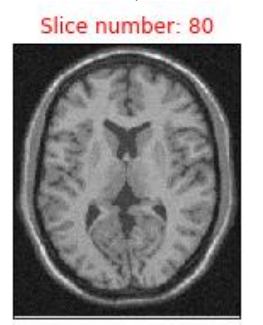

(c) After Bias Correction

Fig 2. N4 Bias Correction

of $15 \times 15 \times 15$ the patch size of the label is also the same. The patches are normalized with zero mean and unit variance before feeding them to the model.

\section{CNN Architecture}

The segmented volumes of the MRI mentioned above are the inputs to the CNN architecture. Fig. 3 gives a detailed overview and Table. I contain the parameters of the CNN model used in this paper. The network consists of 9 layers including the classification layer. The first and second layers consist of 25 feature maps, third and fourth layers have 50 features maps and the fifth layer has 75 feature maps. The layers 6, 7, and 8 are fully connected layers with 400, 200, and 150 feature maps respectively. The classification layers consist of 3 classes GM, WM, and CSF. There are no pooling layers used in this network, hence a unit stride is used in all the convolutional layers. The kernel size of $3 \times 3 \times 3$ for the convolution layers and $1 \times 1 \times 1$ for the fully connected layers is used in order to add more depth in the proposed model. Instead of using the famous ReLU activation function, Parametric Rectified Linear Unit (PReLU) [12] was used in the model and tested. The model with PReLU performed significantly better than the former with ReLU. The PReLU function performs better because of its adaptive property to the given input at a minuscule computational cost. The softmax function is used for calculating the probabilities of the classes and then the argmax function is used to retrieve the class with a maximum probability value. The model was trained for 20 epochs. Early stopping is used to avoid the risk of overfitting of the training data. Cross entropy loss function and adam optimization algorithm are used for reducing the cost. The model was trained again by appending the feature maps of the second and fourth layer in the fully connected layers. Even though the process was computationally very complex due to an increase in parameters at the fully connected layers, but the segmentation was more refined. The proposed model was trained on a system with 16 GB RAM and NVIDIA $1050 \mathrm{Ti}$ GPU with 4 GB of memory.

\begin{tabular}{|ccc}
\hline 3DMSnet & Feature Maps & Input Dimensions \\
\hline Layer 1 & 25 & $25 \times 25 \times 25$ \\
Layer 2 & 25 & $23 \times 23 \times 23$ \\
Layer 3 & 50 & $21 \times 21 \times 21$ \\
Layer 4 & 50 & $19 \times 19 \times 19$ \\
Layer 5 & 75 & $17 \times 17 \times 17$ \\
Fully Connected layer 1 & 400 & $15 \times 15 \times 15$ \\
Fully Connected layer 2 & 200 & $15 \times 15 \times 15$ \\
Fully Connected layer 3 & 150 & $15 \times 15 \times 15$ \\
Classification Layer & 3 & $15 \times 15 \times 15$ \\
\end{tabular}

\section{TABLE I: CNN PARAMETERS}

\section{Reconstructing patches}

Since the classification is done patch wise, hence the patches retrieved after the classification needs to be reconstructed to get the labeled MRI image. The reconstruction process involves aligning the labeled volume in the correct position considering the dimensions of the original image. The patches retrieved from the classification layer are $15 \times 15 \times 15$ which is then appended at the corresponding positions without disturbing the affine co-ordinates and orientation. This 


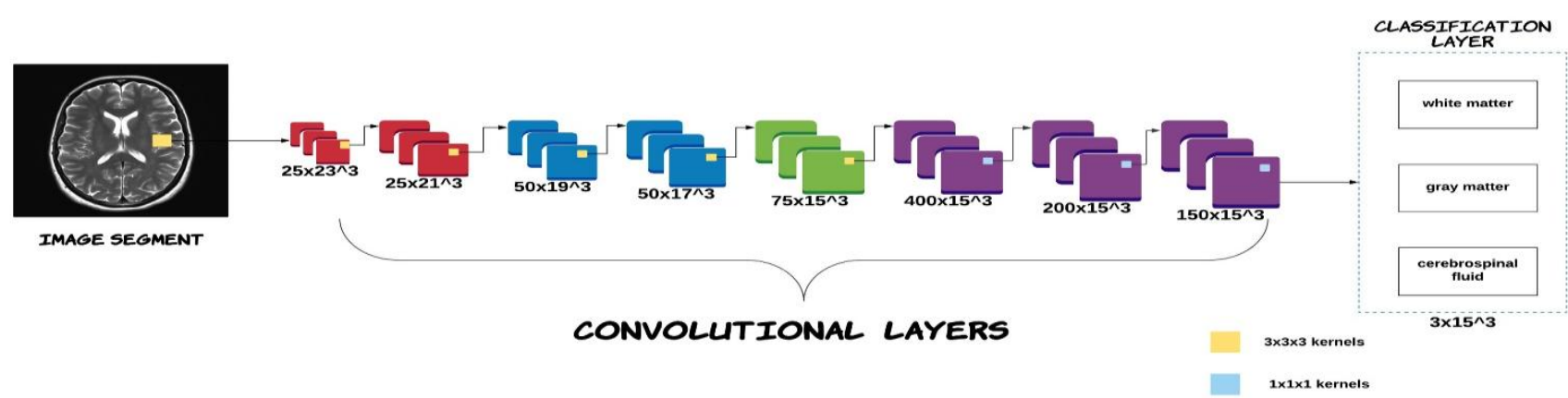

Fig 3. 3DMSnet: Proposed CNN Architecture

process doesn't require rigid/non-rigid registration as the spatial alignment is maintained by the proposed model. The reconstructed image is then compared with the ground truth image to check the accuracy and purity of segmentation.

\section{RESULTS AND EVALUATION}

The trained model was tested on the simulated BrainWeb dataset and some other real datasets publicly available. The testing was conducted on three versions of the same model. Initially, the model was trained using the ReLU activation function. The second version of the model was trained with PReLU as the activation function and the 3DMSnet (final version) using the multi-scale approach. The 3DMSnet performed the best on the test images at the cost of computational complexity. Fig. 4 displays the plot of the segmented image slices achieved from 3DMSnet. The three classes GM, WM and CSF are given different intensity values to distinguish them. The CSF value is merged with the background value. The proposed model outperforms several state-of-the-art segmentation methods with almost none or negligible nearest neighbor errors. The spatial consistency is maintained with the use of the multi-scale approach in the final model.

$$
\rho_{i}=\frac{2 \mid S_{i} \cap G_{i} \|}{\left\|S_{i}+G_{i}\right\|}
$$

The Dice Similarity Coefficient (DSC) [13] is calculated to examine the accuracy of segmentation. The DSC is calculated by overlapping the ground truth with the segmented data. The $S_{i}$ denotes the segmented image and the $G_{i}$ denotes the ground truth data. The $i$ denotes the class in which the pixel is segmented. The DSC is calculated for the

TABLE II: DICE SIMILARITY COEFFICIENT

\begin{tabular}{ll}
\hline Class & Avg. DSC \\
\hline WM & 0.898 \\
GM & 0.810 \\
CSF & 0.944 \\
\hline
\end{tabular}

three classes and the average value of all the $i$ is considered to be the similarity index of the segmented image. The DSC is calculated on 12 MRI test images and average is calculated which is displayed in Table. II. The average of all the three classes is 0.884 which makes the proposed method better than many state-of-art methods.

\section{DISCUSSIONS}

We used the patch-based 3D CNN approach for segmenting GM, WM, and CSF as the main constituent parts of the brain. The use of 3D convolutional layers makes the process little complex but delivers better results over fast and inaccurate results. We tested three versions of our model out of which the multiscale model performs the better than the other two models. The other two models also perform in close proximity with the multiscale model and are computationally less complex than the latter. The small kernel size helps us in getting a deep network and hence getting better details of the features. The model can be tested on any database as the spatial alignment is preserved by the multiscale approach.

\section{FUTURE SCOPE}

This proposed method can have a significant impact on the ongoing research on MRI segmentation. The model can also be used to segment other cortical regions of the brain. The accuracy can be further improved by the use of better data preprocessing techniques. The training period can be significantly enhanced by the use of higher end GPU with huge processing strength. Since there is a rapid increase in brain diseases in the infants, the need for MRI analyzing technology is also growing rapidly.

\section{ACKNOWLEDGMENT}

We would like to take an opportunity to thank our guide $\mathrm{Mr}$. Ajay Kumar for his support and motivation due to which this project is a success. We would extend our hearty thanks to our coordinators Dr. J. Arunnehru and Mrs. G. Chamundeeswari for extending their support in writing this paper. We would also like to thank our colleagues for their support and critical appreciation throughout the project. 

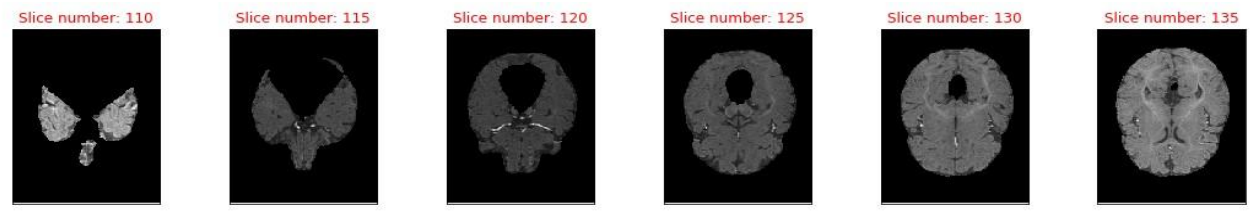

(a) Test Image
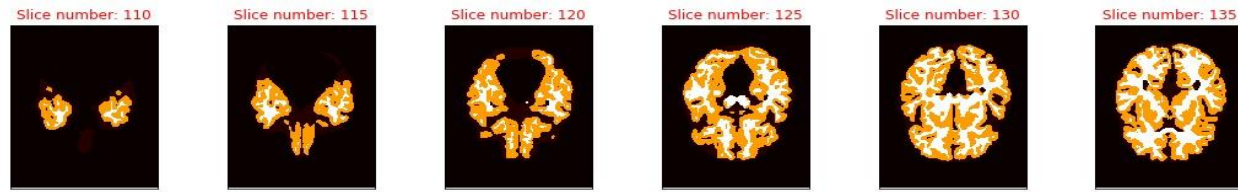

(b) Segmented Image
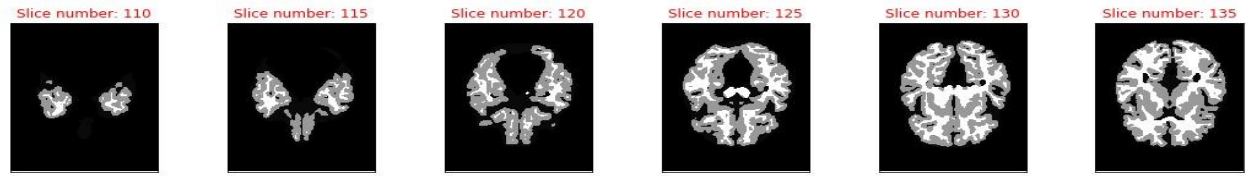

(c) Ground Truth

Fig. 4. Samples of the MRI slices segmented by 3DMSnet

\section{REFERENCES}

1. D.L. Pham, C. Xu, and J.L. Prince, Current methods in medical image segmentation, Annual Review of Biomedical Engineering, vol.2, no.2000, pp.315337,2000

2. N. Passat, C. Ronse, J. Baruthio, J.-P. Armspach, C. Maillot, and C. Jahn, Region-growing segmentation of brain vessels: an atlas based automatic approach, Journal of Magnetic Resonance Imaging, vol.21, no.6, pp.715725,2005

3. T. H. Lee, M. F. A. Fauzi and R. Komiya, "Segmentation of CT Brain Images Using K-Means and EM Clustering,” 2008 Fifth International Conference on Computer Graphics, Imaging and Visualization, Penang, 2008, pp. 339-344.

4. C. Wan, M. Ye, C. Yao and C. Wu, "Brain MR image segmentation based on Gaussian filtering and improved FCM clustering algorithm," 2017 10th International Congress on Image and Signal Processing, 2017, pp. 1-5.

5. A. Gholipour, N. Kehtarnavaz, R. Briggs, M. Devous and K. Gopinath, "Brain Functional Localization: A Survey of Image Registration Techniques," in IEEE Transactions on Medical Imaging, vol. 26, no.

6. 4, pp. 427-451, April 2007.

7. N. Torbati and A. Ayatollahi, "A new method for non-rigid registration of MRI images," 2017 Iranian Conference on Electrical Engineering (ICEE), Tehran, 2017, pp. 91-94.

8. N. H. Rajini and R. Bhavani, "Classification of MRI brain images using k-nearest neighbor and artificial neural network," 2011 International Conference on Recent Trends in Information Technology (ICRTIT), Chennai, Tamil Nadu, 2011, pp. 563-568.

9. P. Moeskops, M. A. Viergever, A. M. Mendrik, L. S. de Vries, M. J.

10. N. L. Benders and I. Igum, "Automatic Segmentation of MR Brain Images With a Convolutional Neural Network," in IEEE Transactions on Medical Imaging, vol. 35, no. 5, pp. 1252-1261, May 2016.

11. McConnell brain imaging center, BrainWeb: simulated brain database, http://www.bic.mni.mcgill.ca/brainweb/.

12. N4ITK: improved N3 bias correction IEEE transactions on medical imaging vol. 29,6 (2010): 1310-20.

13. K. Kamnitsas, C. Ledig, V. F. Newcombe, J. P. Simpson, A. D. Kane, D. K. Menon, D. Rueckert, B. Glocker, Ecient multi-scale 3D CNN with fully connected crf for accurate brain lesion segmentation, arXiv preprint arXiv:1603.05959.

14. K. He, X. Zhang, S. Ren and J. Sun, "Delving Deep into Rectifiers: Surpassing Human-Level Performance on ImageNet Classification,"

15. 2015 IEEE International Conference on Computer Vision (ICCV), Santiago, 2015, pp. 1026-1034. doi: 10.1109/ICCV.2015.123

16. L. R. Dice, Measures of the amount of ecologic association between species, Ecology, vol.26, no.3, pp.297302, 1945 BioMedical Engineering and Informatics (CISP-BMEI), Shanghai,

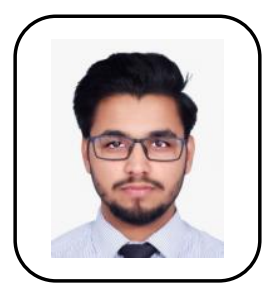

\section{Authors Profile}

Junaid Ahmad is an undergraduate student at SRM Institute of Science and Technology. His research interest lies in the field of Computer Vision and Machine Learning. He has recently received a funded admission offer from University of Alberta for master's program.

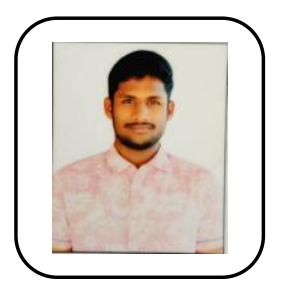

Bhanu Bhaskar is an undergraduate student at SRM Institute of Science and Technology. His research interest lies in the field of Intelligent agents and autonomous systems. He is persistent in his intent to pursue higher education in his field of interest and perform quality research work.

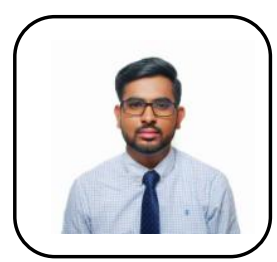

Haresh Seetharaman is an undergraduate student at SRM Institute of Science and Technology. He currently works as a Software Developer at Siemens Healthcare Ltd. His major research interest lies in the field of medical image analysis using AI and Computer Vision Technology.

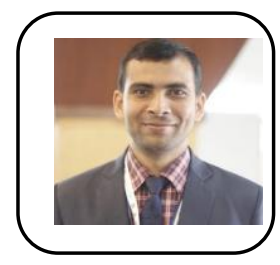

Ajay Kumar is an Assistant Professor at SRM Institute of Science and Technology. His research interests are Design and Algorithm analysis and Object-Oriented Programming.

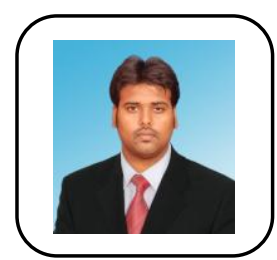

J. Arunnehru is an Assistant Professor at SRM Institute of Science and Technology. His research interests are Computer Vision, Pattern Recognition and Image Processing. He completed his PhD on Pattern Recognition and Machine Learning.

\section{Published By:}

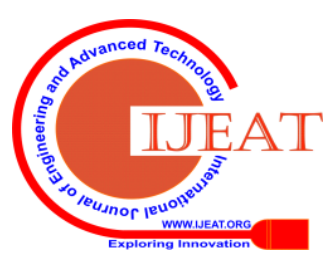

